This document is confidential and is proprietary to the American Chemical Society and its authors. Do not copy or disclose without written permission. If you have received this item in error, notify the sender and delete all copies.

\title{
Investigation of Many-body Exciton Recombination and Optical Anisotropy in Two-dimensional Perovskites having Different Layer with Alternating Cations in the Interlayer Space
}

\begin{tabular}{|r|l|}
\hline Journal: & The Journal of Physical Chemistry \\
\hline Manuscript ID & jp-2021-013185.R1 \\
\hline Manuscript Type: & Article \\
\hline Date Submitted by the & $19-$ Mar-2021 \\
\hline Complete List of Authors: & $\begin{array}{l}\text { Ghosh, Supriya; Jacobs University Bremen gGmbH, Physics and Earth } \\
\text { Sciences } \\
\text { Pradhan, Bapi; Katholieke Universiteit Leuven Faculteit Wetenschappen, } \\
\text { Chemistry } \\
\text { Zhang, Yiyue; Katholieke Universiteit Leuven Faculteit Wetenschappen, } \\
\text { Chemistry } \\
\text { Rana, Debkumar; Jacobs University Bremen gGmbH, Physics and Earth } \\
\text { Science } \\
\text { Naumenko, Denys; Graz University of Technology } \\
\text { Amenitsch, Heinz; Technische Universitat Graz, } \\
\text { Hofkens, Johan; Katholieke Universiteit Leuven Faculteit Wetenschappen, } \\
\text { Chemistry; Max Planck Institute for Polymer Research, } \\
\text { Materny, Arnulf; Jacobs University Bremen gGmbH, Physics and Earth } \\
\text { Sciences }\end{array}$ \\
\hline
\end{tabular}

\section{SCHOLARONE Manuscripts}


Investigation of Many-body Exciton Recombination and Optical Anisotropy in Twodimensional Perovskites having Different Layer with Alternating Cations in the Interlayer Space

Supriya Ghosh', Bapi Pradhan ${ }^{\S}$, Yiyue Zhang ${ }^{\S}$, Debkumar Rana ${ }^{\dagger}$, Denys Naumenko, ${ }^{\#}$ Heinz Amenitsch, ${ }^{\#}$ Johan Hofkens ${ }^{\S, \delta}$, and Arnulf Materny ${ }^{\dagger *}$

${ }^{\dagger}$ Department of Physics and Earth Sciences, Jacobs University Bremen, Campus Ring 1, 28759 Bremen, Germany

${ }^{\S}$ Department of Chemistry, KU Leuven, Celestijnenlaan 200F, 3001 Heverlee, Belgium

"Institute of Inorganic Chemistry, Graz University of Technology, Stremayrgasse 9/V, Graz, 8010, Austria

${ }^{\delta}$ Max Planck Institute for Polymer Research, Ackermannweg 10, 55128 Mainz, Germany

\author{
AUTHOR INFORMATION \\ Corresponding Authors \\ *a.materny@jacobs-university.de +49 421 200-3231
}




\begin{abstract}
Two-dimensional (2D) perovskites with alternating cations in the interlayer space (ACI) represent a new type of structure with different physical properties compared to the more common Ruddlesden-Popper (RP) counterparts. Still, there is a lack of understanding of photophysical and optoelectronic properties. In our work, we have used transient absorption spectroscopy to get better insight into the nature of photoexcitations in ACI perovskites with varying layers. We observed that multiple exciton recombination predominates, compared to self-trapping of excitons, with increasing the number of layers (expressed by $n$ ). With decreasing $n$, the ACI perovskite shows less many-body exciton recombination due to a very fast self-trapping of the excitons. Furthermore, we observed that the optical anisotropy increases with increasing number of the inorganic layers as the polarization orientation increases. Comparing the photophysical properties of the three different systems, we suggest that ACI3 is most promising for photovoltaic and optical polarization devices.
\end{abstract}

\title{
TOC Image
}

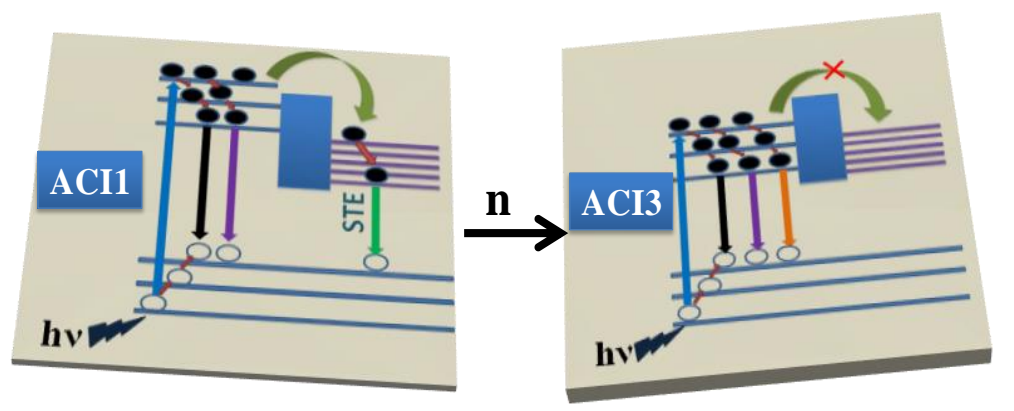




\section{Introduction}

The last few years have witnessed a dramatic development of metal halide perovskite (MHP) materials for optoelectronic applications. ${ }^{1-5}$ MHPs are promising candidates for next-generation light-emitting diodes (LEDs) due to their sharp emission peaks, high luminescence quantum yields (QY), and easy and low-cost solution-processable synthesis. ${ }^{6-10}$ To enhance device performance, intense efforts were made in recent years for developing new perovskite materials and device architectures. Here, much work has been devoted to gain a better understanding of the working mechanisms following an optical excitation. ${ }^{11-14}$ By suppressing nonradiative recombination and balancing carrier injection, the efficiency of the devices (particularly lightemitting and photovoltaic devices) has been significantly improved. ${ }^{13,15-17}$ Despite a significant increase in efficiency, the development of 3D-perovskite-based devices still suffers from their poor stability. ${ }^{18-20}$ Firstly, because of their soft structure, they can easily decompose in a hot or humid environment. Secondly, organic cations and halides can move under electric field, giving rise to instability of the perovskite structure. ${ }^{21-23}$ These problems have to be overcome in order to make the devices suitable for real applications. Two-dimensional (2D) structures have emerged as a possible solution to these issues. Presently, they are considered as promising material with significant potential for optoelectronic applications. ${ }^{24}$

Among the layered 2D families, the Ruddlesden-Popper (RP) organic-inorganic halide perovskites are by far the most common type. They have attracted attention since they seem to provide long-term stability for perovskite solar cells. ${ }^{25-27}$ Other variations, including the DionJacobson (DJ) structure, have also emerged in the halide perovskite family. ${ }^{28-30}$ The less common DJ type is chemically different from the RP variant in that the two spacer cations, $(M)_{2}$, of the $(M)_{2}(A)_{n-1} B_{n} X_{3 n+1} R P$ structure are replaced by a single alkali metal $M^{\prime}$ spacer in the $\left(M^{\prime}\right)(A)_{n-1} B_{n} X_{3 n+1}$ DJ structure. In their recent work, Kanatzidis and co-workers ${ }^{19}$ have introduced hybrid 2D lead iodide perovskites with two different alternating cations in the 
interlayer space (ACI), derived from oxide perovskites. The ACI perovskites are described by the formula $(\mathrm{GA})(\mathrm{MA})_{\mathrm{n}} \mathrm{Pb}_{\mathrm{n}} \mathrm{I}_{3 \mathrm{n}+1}(\mathrm{GA}=$ guanidinium, $\mathrm{MA}=$ methylammonium $)$, and they are stabilized by the ordering of two different cations (GA and MA) in the interlayer space. ${ }^{19}$ This arrangement results in closer contacts of the $\left[\mathrm{Pb}_{n} I_{3 n+1}\right]$ unit. Compared to the more common RP 2D perovskites, the ACI perovskites have a different stacking arrangement and show higher crystal symmetry and different physical properties. From an application point of view, these ACI perovskites are very interesting since they show a high potential as light-harvesting materials for solar cells, with a power conversion efficiency (PCE) of $\sim 18 \%$ and a high opencircuit voltage of $\sim 1 \mathrm{~V}$ recorded for $(\mathrm{GA})(\mathrm{MA})_{\mathrm{n}} \mathrm{Pb}_{\mathrm{n}} \mathrm{I}_{3 \mathrm{n}+1}(n=3)$ based solar cells. ${ }^{19,31-32}$

For the RP perovskites, a considerable number of studies has been performed contributing to a detailed understanding of photophysical processes and their impact on device performance. ${ }^{33-}$ ${ }^{34}$ On the other hand, for the hybrid ACI perovskites, there have been only a few reports on the investigation of the processes following photo excitation. ${ }^{31}$ A detailed understanding of exciton trapping, recombination and interaction processes for varying 2D layers is missing. This information is necessary for the improvement of the photovoltaic and light-emission performance of the $2 \mathrm{D}$ perovskite-based devices. Therefore, the goal of our work is to learn more about the nature of excitons in these systems, which is important for both fundamental understanding and potential applications.

In the following, we present our investigation of the charge-carrier dynamics in ACI perovskite with varying layers and excitation densities. For the time-resolved experiments, we have used transient absorption and photoluminescence spectroscopy as well as transient anisotropy measurements. From the results valuable insights into the light-driven processes contributing to a better device design for ACI perovskites in the future can be gained. 


\section{Experimental Section}

\section{Synthesis of ACI Perovskite}

Chemicals: Lead iodide $\left(\mathrm{PbI}_{2}, 99.99 \%\right.$; Alfa Aesar), methylammonium iodide (MAI, 99\%, Great cell Solar Materials), guanidine iodide (GAI, 99\%, Great cell Solar Materials), Toluene (anhydrous, 99.8\%, Merck) and N, N-dimethylformamide (DMF, anhydrous, 99.8\%, SigmaAldrich) were purchased and used as is. The main stock of the chemicals was stored in a $\mathrm{N}_{2}$-filled glovebox to prevent the influence of moisture and oxygen.

Preparation of Thin Films: Stoichiometric ratios of the precursors of MAI, GAI, and $\mathrm{PbI}_{2}$ were dissolved in a mixed solvent of DMF and DMSO (volume ratio of 9:1) to obtain $1.2 \mathrm{M}$ $\mathrm{GAMA}_{n} \mathrm{~Pb}_{n} \mathrm{I}_{3 \mathrm{n}+1}(n=1,2$, and 3$)$ precursor solutions. ${ }^{31}$ The solutions were stirred at room temperature overnight to achieve complete dissolution in the mixed polar solvents. $2.5 \times 2.5 \mathrm{~cm}^{2}$ glass substrates were cleaned by sequential sonication in soap water, acetone, isopropanol, and ethanol for $30 \mathrm{~min}$ each and then dried under air flow followed by ozone plasma treatment for 15 min. All the films were prepared under inert atmosphere inside a $\mathrm{N}_{2}$-filled glovebox. Asprepared solutions were spin-coated onto the glass substrates in two steps with $500 \mathrm{rpm}$ for $3 \mathrm{~s}$ followed by $4000 \mathrm{rpm}$ for $60 \mathrm{~s}$. Towards the end of the spin-coating process ( $45 \mathrm{~s}), 200 \mu \mathrm{L}$ of chlorobenzene were dropped onto the substrate followed by annealing of the films at $80{ }^{\circ} \mathrm{C}$ for $15 \mathrm{~min}$.

\section{Spectroscopic Techniques}

Absorption and PL. For all optical measurements, the particles dispersed in hexane were dropcasted on a cover slip followed by drying at room temperature. Absorption and PL spectra were taken using a Perkin Elmer Lambda 950 and an Edinburgh FLS980 spectrometer, respectively.

Time-resolved PL. The lifetimes of the drop-casted thin-films on cover slides were probed by using a confocal fluorescence microscope (Leica TCS SP8 X). A $470 \mathrm{~nm}$ laser emitting pulses 
with $2.5 \mathrm{MHz}$ repetition rate has been used for all ACI perovskites, studying the PL and its decay dynamics. The PL was detected using a fast hybrid photodiode and the inbuilt software has been used to fit the exponential decays.

Femtosecond Transient Absorption (TA) Spectroscopy. The femtosecond TA technique used in this work has been previously described elsewhere. ${ }^{35-37}$ In brief, a Ti:sapphire regenerative amplifier (CPA 2010, Clark MXR) seeded by an oscillator was used as light source. The laser output from the amplifier had a central wavelength of $\sim 800 \mathrm{~nm}$, and an energy of $1 \mathrm{~mJ}$ per pulse at a repetition rate of $1 \mathrm{kHz}$. This laser output was split by a 50:50 beam splitter into two beams which pumped two optical parametric amplifiers (OPAs) (TOPAS, Light Conversion) capable of producing different laser wavelengths from UV to near infrared. In order to correct for the chirp introduced by the group velocity dispersion (GVD) in the different optical components, the output of each TOPAS was re-compressed using a standard two-pass prism-compressor setup, resulting in nearly Fourier-transform limited femtosecond pulses of about $80-90$ fs pulse widths. One TOPAS was used for producing the pump pulses and the other one for generating the probe pulses. Pump and probe pulses were then focused and spatially overlapped in the sample. The timing between the pump and the probe pulses was achieved by using computer-controlled translation delay stages in a Michelson-type setup, which allowed for up to about 500 ps time delay with a resolution clearly less than the crosscorrelation of pump and probe laser pulses. Following the initial photoexcitation of the sample by the pump pulse, the time-delayed probe pulse transmission through the sample was detected by a photodiode in order to monitor the dynamics of the photogenerated species in the sample. A boxcar amplifier was used for pulse averaging in order to optimize the signal-to-noise ratio.

A white light continuum (WLC) probe in the visible wavelength range was generated by sending a small fraction of the $800 \mathrm{~nm}$ focused beam through a sapphire crystal. In order to obtain stable WLC, an iris and neutral density filters were used for adjusting the intensity of the 
$800 \mathrm{~nm}$ light. Pump and probe pulses were spatially overlapped at the sample position. To eliminate low-frequency laser noise, the probe was split into two beams and detected as sample and reference separately. The detection of the transmitted probe light was performed separately with the pump blocked and unblocked, which was achieved using a mechanical chopper with $500 \mathrm{~Hz}$ rotational frequency. The TA spectra were recorded by CCD arrays after dispersion using a grating spectrograph (Acton spectra Pro SP 2358).

Grazing-Incidence Wide-Angle X-Ray Scattering (GIWAXS). GIWAXS measurements have been performed at the Austrian SAXS beamline at the ELETTRA synchrotron (Trieste, Italy) at a photon energy of $8 \mathrm{keV} .{ }^{38}$ The beam size was set to $1 \mathrm{x} 0.1 \mathrm{~mm}^{2}(\mathrm{HxV})$. The sampleto-detector (Pilatus3 1M, Dectris) distance was adjusted to $217.3 \mathrm{~mm}$ after calibration with silver behenate as a reference pattern. All measurements have been performed at an incident angle of $0.5^{\circ}$ in air. The patterns have been corrected for the fluctuations of the primary intensity. ${ }^{39}$ The reconstruction of the GIWAXS patterns and three-dimensional structure indexing have been performed with the GIXSGUI toolbox. ${ }^{40}$ The GIWAXS patterns shown in Figure 1d-f are represented by orthogonal $q_{\mathrm{z}}$ and $q_{\mathrm{r}}$ reciprocal space axes, where $q_{\mathrm{r}}$ is a total inplane wave vector transfer, and $q_{\mathrm{z}}$ is a wave vector transfer component normal to the surface. Note that in such representation a missing wedge appears due to a fact that the intensity distribution on an area detector measures the projection of the reciprocal lattice intercepted by the Ewald sphere onto the detector plane. The structure indexing is performed according to the adjusted unit cell parameters given in Table S1. 


\section{Results and Discussion}

The crystal structures of the ACI perovskites are illustrated in Scheme 1; all three compounds form crystals with an orthorhombic space group. ${ }^{19}$ Optical absorption measurements yield information about the distribution of the various phases defined by the number of layers $(n)$ and the presence of internal photoexcited charge transfer. Figure 1a and $1 \mathrm{~b}$ depicts the steady state and transient absorption spectra of the ACI perovskite for $n=3$ while panels (a) and (b) of Figure S1 show the spectra for $n=1$ and 2, respectively. In the following, we refer to the samples with monolayer $(n=1)$, bilayer $(n=2)$, and trilayer $(n=3)$ as ACI1, ACI2, and ACI3, respectively. The spectra exhibit a red shift of the band gap with increasing layer thickness starting from $E_{g}=2.11 \mathrm{eV}$ for ACI1 to $1.99 \mathrm{eV}$ for ACI2, and to $1.90 \mathrm{eV}$ for ACI3. This is also reflected in the color of the compounds, which changes from red (ACI1) to dark red (ACI2), and finally to "black" (ACI3). The photoluminescence (PL) spectra follow a similar trend like the absorption spectra. ACI1, ACI2, and ACI3 exhibit PL peaks at 700, 752, and $780 \mathrm{~nm}$, respectively (Figure 1c). The crystal structure of ACI perovskite films have been investigated by grazing-incidence wide-angle X-ray scattering (GIWAXS) measurements (Figure 1d-f). The films have been characterized above the critical angle to provide a depth-sensitive information with the X-ray beam penetrating into the film. The indexing of the diffraction spots has been performed in order to reveal the crystal structure and the crystal orientation relative to the substrate. ${ }^{40}$ The lattice constants obtained on single ACI crystals ${ }^{19}$ have been adjusted (Table S1) to match the prominent reflections in GIWAXS patterns as depicted in Figure 1d-f. In particular, peaks at $q_{z}=0.65 \AA^{-1}$ (ACI1) and $q_{z}=0.85 \AA^{-1}$ (ACI2 and ACI3) are observed along the out-of-plane scattering direction, which have been assigned to (002), (004), and (006) reflections of $2 \mathrm{D}$ perovskites, testifying that crystals are preferentially oriented with their $\mathrm{c}$ axis perpendicular to the substrate for all films. The ACI1 film shows the presence of a $\mathrm{MAPbI}_{3}$ phase $\left(q_{z}=0.45 \AA^{-1}\right.$ and $\left.q_{z}=0.8 \AA^{-1}\right)$ presumably due to its photo-instability. ${ }^{31}$ With increasing 
dimensionality (from $n=1$ to 3 ), the intensity of the diffraction spots over the diffuse diffraction rings increases, implying that ACI3 is more ordered than ACI1 and ACI2.

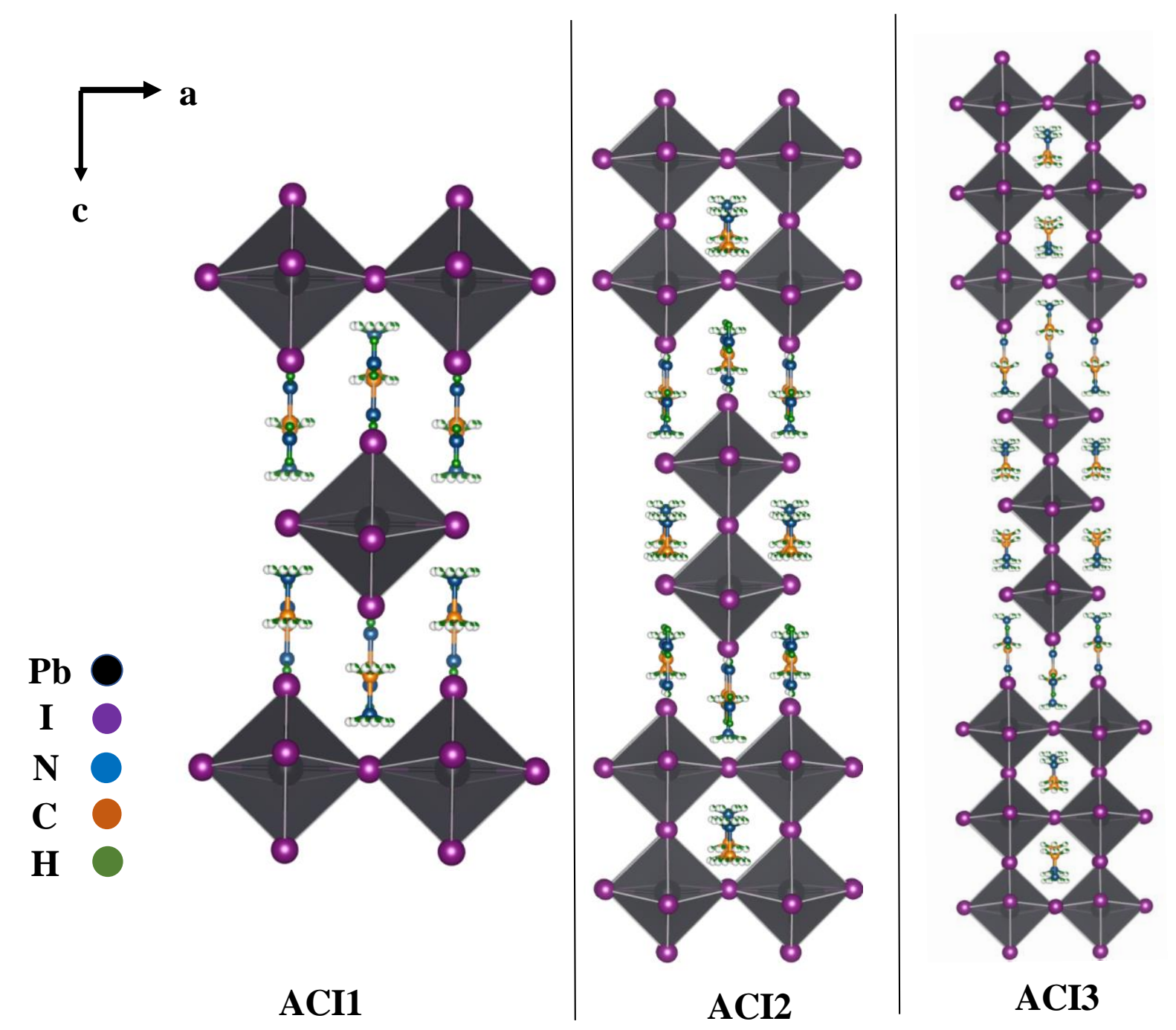

Scheme1. View of the unit cells of the ACI1, ACI2 and ACI3 perovskites along the crystallographic b-axis.

The increased full width at half maximum (FWHM) of the PL band seen for ACI1 (Figure 1c; the fitting result using a Voigt profile is shown in Figure S2) indicates the higher phonon scattering effects. ${ }^{33,41}$ It is important to understand that the nature of the emitting states is connected with carrier-phonon couplings. ${ }^{33}$ According to previous reports, a strong carrierphonon interaction has been invoked to account for below-band-gap trap states and broad 
emissions in 2D layer perovskites. ${ }^{14,42-43} \mathrm{Wu}$ et al. ${ }^{14}$ and others ${ }^{44-45}$ describe that self-trapping of excitons results from exciton-phonon interactions, which depend on the dimensionality of a crystalline system. Self-trapping of excitons becomes more prominent with decreasing number of $n$, since the reduction of the dimensionality of a crystalline system lowers the deformation energy, facilitating self-trapping. ${ }^{14,42,45-46}$ It is important to note that at room temperature free exciton (FE) recombination is comparable with STE. ${ }^{14,42-43,45}$ The FWHM values for ACI1, ACI2, and ACI3 are 200, 140, and $100 \mathrm{meV}$ respectively. From this, it can be attributed that the density of self-trapped excitons is decreasing from ACI1 over ACI2 to ACI3.
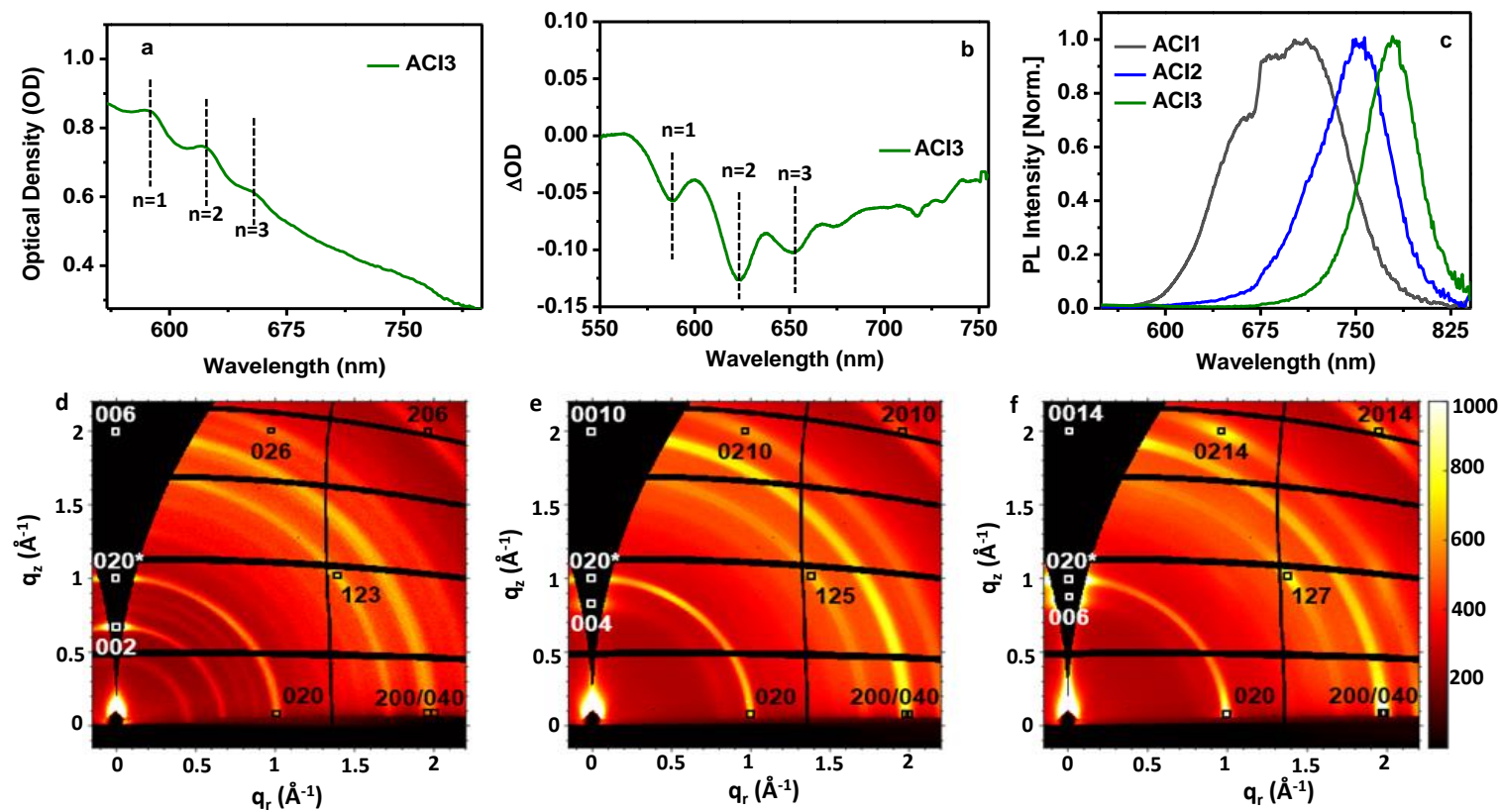

Figure 1. (a) Absorption and (b) transient absorption (TA) spectrum of ACI3 perovskites. (c) Steady-state PL spectra of ACI1 (black line), ACI2 (blue line), and ACI3 (green line) perovskites. GIWAXS patterns of (d) ACI1, (e) ACI2, and (f) ACI3 perovskite films acquired at an incidence angle of $0.5^{\circ}$.

Besides having an increased line width, the PL band seen for ACI1 is more asymmetric compared to those of ACI2 and ACI3. The broad and asymmetric PL line shape of ACI1, which 
also shows a sub-structure, points to the coexistence of multiple emissive states (both FE and STE), as reported in literature, ${ }^{8,33}$ which is decreasing with increasing number $n$. An assignment of the features seen for the PL band of ACI1 to specific contributions is not possible at the moment, since temperature-dependent PL measurements would be needed for that, ${ }^{14,42-43}$ which will be a task for our future studies.

Figure 2a shows the decay dynamics of the PL (monitored at peak maxima) from ACI1 (black line), ACI2 (blue line), and ACI3 (green line) following $470 \mathrm{~nm}$ excitation. It is clear from the figure that the PL decay gets faster with decreasing number of layers. The decay dynamics can be described by a multiexponential function to fit the experimental data. From these multiexponential fits, we have calculated an average PL lifetime $\left(\tau_{\text {average }}\right)$, which is decreasing from $14.1 \mathrm{~ns}$ for ACI1 to $8.2 \mathrm{~ns}$ for ACI2, and to $2.7 \mathrm{~ns}$ for ACI3. The longer PL lifetime found for the ACI1 sample can be explained by the increased contribution of self-trapped exciton (STE) recombination. With increasing layer, the activation energy barrier between FE and STE increases and consequently the contribution of STE decreases and FE increases (see Table S2). 

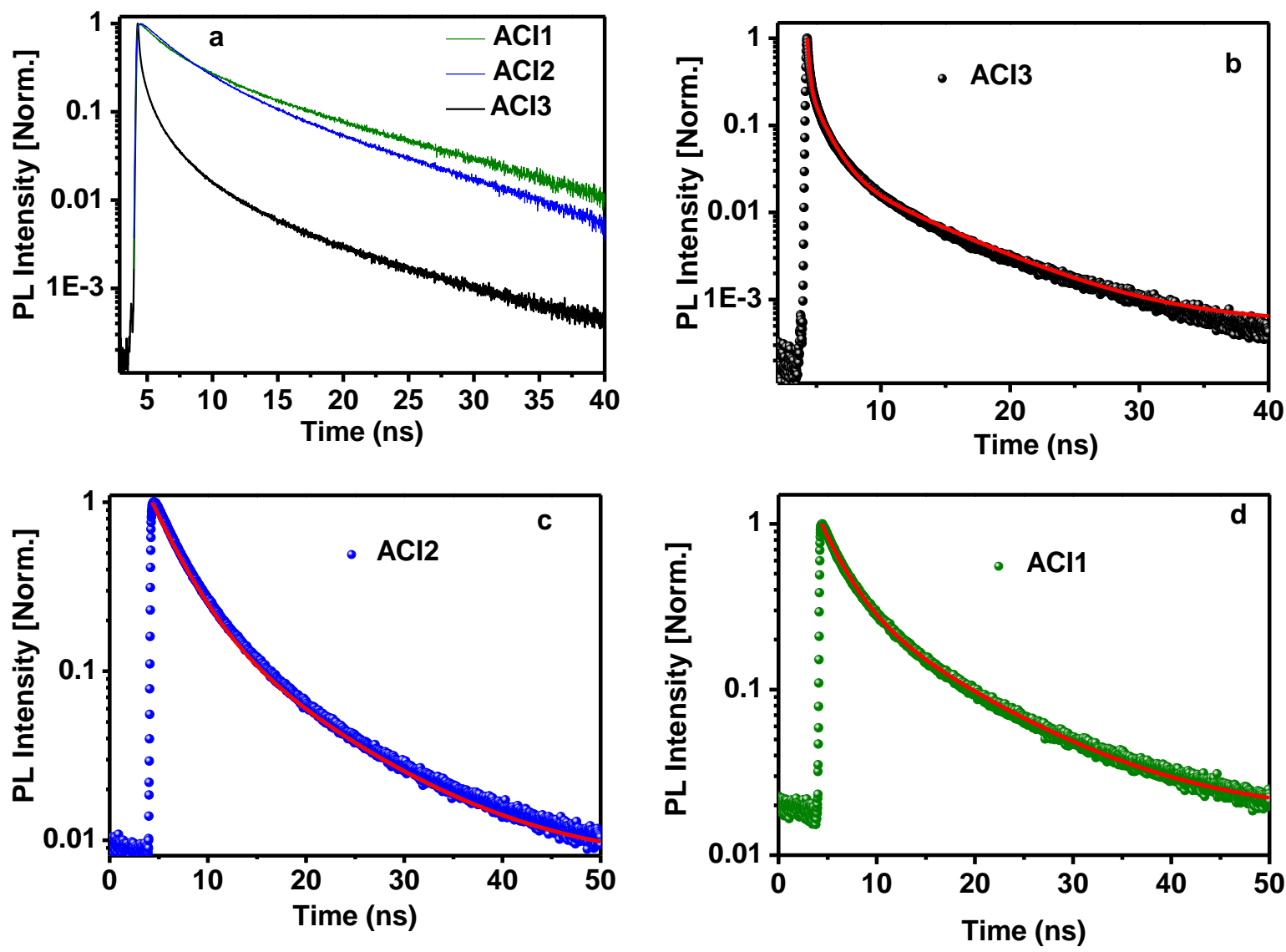

Figure 2. (a) Comparison of PL decay profiles of ACI1 (green line), ACI2 (blue line), and ACI3 (black line). The excitation wavelength is $470 \mathrm{~nm}$ and the emission peak wavelengths are 700, 752, and $780 \mathrm{~nm}$ for ACI1, ACI2, and ACI3 perovskites, respectively. The fitting results (red lines) are shown for the PL decay dynamics of (b) ACI1, (c) ACI2, and (d) ACI3.

The relatively low temporal resolution of the time-dependent PL measurements did not allow for accessing several ultrafast processes like carrier trapping, different order exciton recombination processes, etc. In order to also gain information about these processes, we have performed femtosecond time-resolved transient absorption (TA) measurements on the different ACI perovskite samples. This mainly enabled us to demonstrate the effect of trap states on the charge-carrier dynamics. Figure $1 \mathrm{~b}$ depicts the TA spectrum of the ACI3 perovskite. Comparing the observed changes in optical density $\triangle \mathrm{OD}$ with the steady-state absorption spectrum, one finds that the dominating features are due to the bleach corresponding to the absorption peaks 
of ACI1, ACI2, and ACI3, in accordance with the results reported earlier. ${ }^{31}$ This bleaching allows us to study important processes at early times by analyzing the TA dynamics at the minima of the TA spectrum, i.e. the maxima of the negative $\Delta \mathrm{OD}$ spectrum (bleaching position) assigned to ACI1, ACI2, and ACI3, selectively (Figure 3a). In each case, the sample was excited above the band gap with a $490 \mathrm{~nm}$ pump laser pulse. The TA dynamics of each sample (Figure $3 \mathrm{~b}-\mathrm{d})$ have been measured at low $\left(4.6 \times 10^{13}\right.$ photons $\left./ \mathrm{cm}^{2}\right)$ and high $\left(2.1 \times 10^{14}\right.$ photons $\left./ \mathrm{cm}^{2}\right)$ excitation pulse intensity, which we label as $I I$ and $I 2$, respectively. For each sample, we have fitted the TA dynamics of both $I 1$ and $I 2$ using simple biexponential functions. In each case, we found a fast ( $>5 \mathrm{ps}$ ) and a slow (>30 ps) time component (see Table S3). The slow component is similar to that reported in literature where it was ascribed to biexciton Auger recombination. ${ }^{13,47}$ The fast time component is most presumably associated with higher-order recombination of excitons.

We found that this fast component contributes more when excited with $I 2$ compared to the case where the smaller excitation intensity $I I$ is used, while, reversely, the slower component dominates for $I I$ excitation. This clearly indicates that the contribution of the biexciton recombination process dominates for low pump intensity. With increasing intensity, the higherorder exciton recombination starts to predominate the biexcitonic one. For a decreasing thickness, i.e. a decreasing number of monolayers, we observed that the fast component is getting slower. This indicates that the self-trapping of excitons is a faster process than that related to higher-order exciton recombination. As the photoexcited carriers depopulate very quickly via self-trapping of excitons, the contribution of higher-order exciton recombination decreases. With increasing $n$, the energy barrier between the FE and STE state is increasing and the higher-order Auger recombination process dominates over the STE process. ${ }^{14}$ 

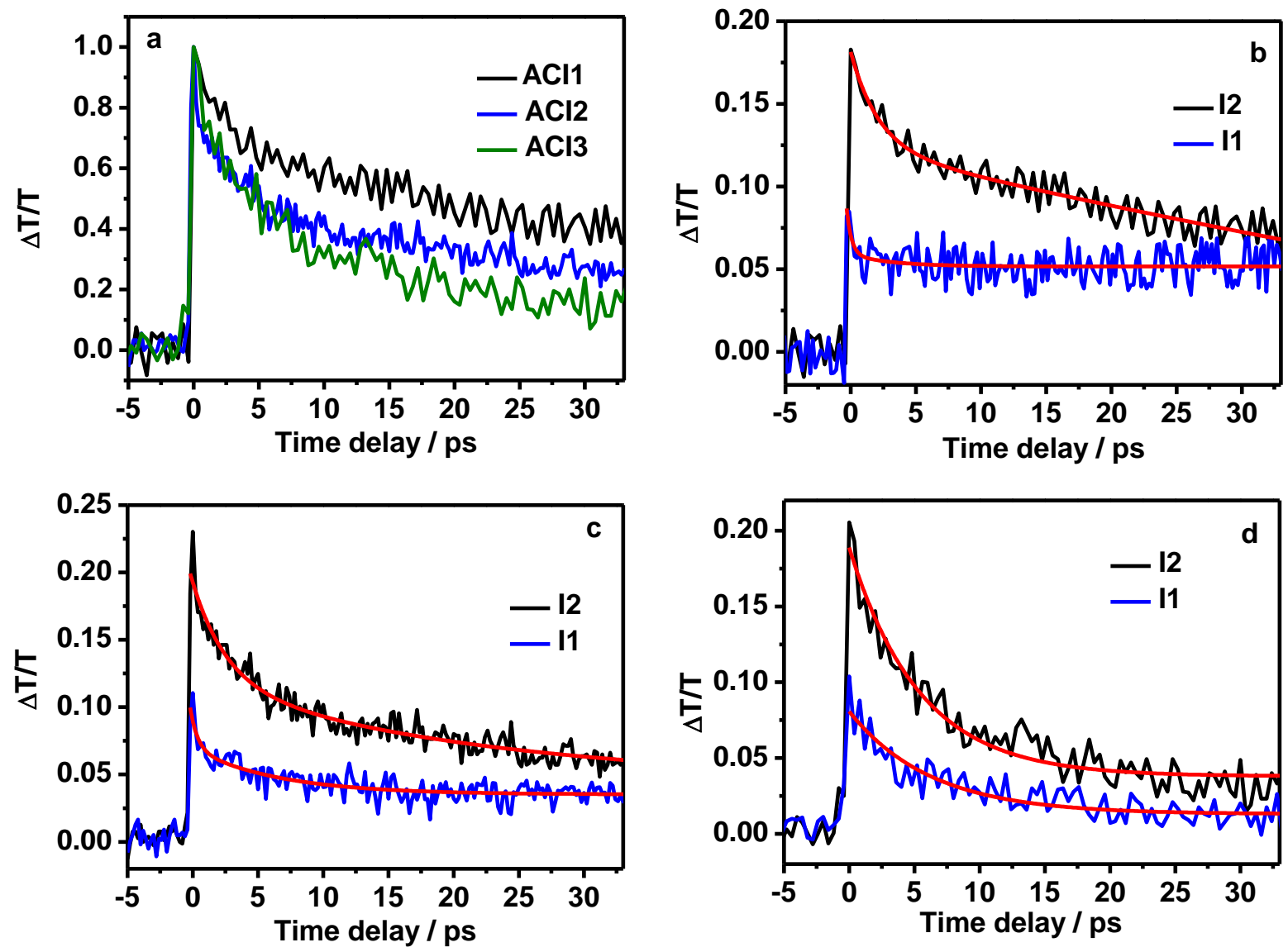

Figure 3. (d) Comparison of the TA dynamics for ACI1 (black), ACI2 (blue), and ACI3 (olive) perovskites. TA dynamics at excitation intensities $I 1$ (blue) and $I 2$ (black) of (b) ACI1, (c) ACI2, and (d) ACI3 perovskites. The solid red lines are the results from fitting the decay curves using equation 1. In the experiments, a pump wavelength of $490 \mathrm{~nm}$ and probe wavelengths of 585,620 , and $650 \mathrm{~nm}$ were used for ACI1, ACI2, and ACI3, respectively.

To address the different orders of the Auger recombination processes, we used many-body exciton recombination models. If the Auger process occurs between two excitons (biexciton), the rate equation can be described as

$\frac{d \rho}{d t}=-k_{1} \rho-k_{2} \rho^{2}$ 
where $\rho$ is the number density of excitons, $k_{1}$ is the first-order rate constant corresponding to single-exciton recombination, and $\mathrm{k}_{2}$ is the second-order rate constant for biexciton recombination. The solution of equation 1 is

$\rho=\frac{B k_{1} e^{-k_{1} t}}{1-B k_{2} e^{-k_{1} t}}$

where $B=\frac{\rho_{0}}{k_{1}-k_{2} \rho_{0}}$ and $\rho_{0}$ is the initial exciton density. Figure $\mathrm{S} 3$ demonstrates that for intensity I1, the TA dynamics of all three ACI sample are fitted well with the biexciton recombination model. However, the experimental data obtained with $I 2$ excitation are not fitted well with equation 2 (Figure S3). This indicates that the biexciton model does not properly describe the population decay at higher excitation densities. The estimated $k_{2}$ from the fitting is found to be $2.7 \pm 0.1 \times 10^{-11} \mathrm{~cm}^{3} \mathrm{~s}^{-1}$ for ACI1

To confirm the higher-order exciton recombination process, we have assumed a three-body exciton recombination model for the temporal evolution of the excitonic bleach of ACI1, ACI2, and ACI3; the rate equation can be described as ${ }^{12}$

$\frac{d \rho}{d t}=-k_{1} \rho-k_{2} \rho^{2}-k_{3} \rho^{3}$

where again $\rho$ is the exciton density, $k_{1}$ the first-order rate constant corresponding to single exciton recombination, $k_{2}$ the second-order rate constant for biexciton recombination, and $k_{3}$ the rate constant describing the third-order Auger recombination. As the first-order recombination rate is much slower than the second- and third-order processes, we have neglected the first term in equation 3. Assuming $k_{1}=0$, equation 3 can be solved as

$t=C+\frac{1}{k_{2} \rho}+\frac{k_{3}}{k_{2}^{2}} \ln \left(\frac{\rho}{k_{2}+k_{3} \rho}\right)$

where $C$ is a constant. Fitting of the TA dynamics corresponding to ACI3 using equation 4 yields a value for $k_{3}$ around $1.1 \pm 0.5 \times 10^{-27} \mathrm{~cm}^{6} \mathrm{~s}^{-1}$. Figures S3 and S4 show that the exciton 
decay dynamics induced by the pump laser with intensity $I 2$ can be described well using this three-body exciton interaction model for all three samples (see Scheme 2 for a presentation of the different processes). At intensity $I 1$, the biexciton recombination becomes competitive with the higher-order exciton recombination.

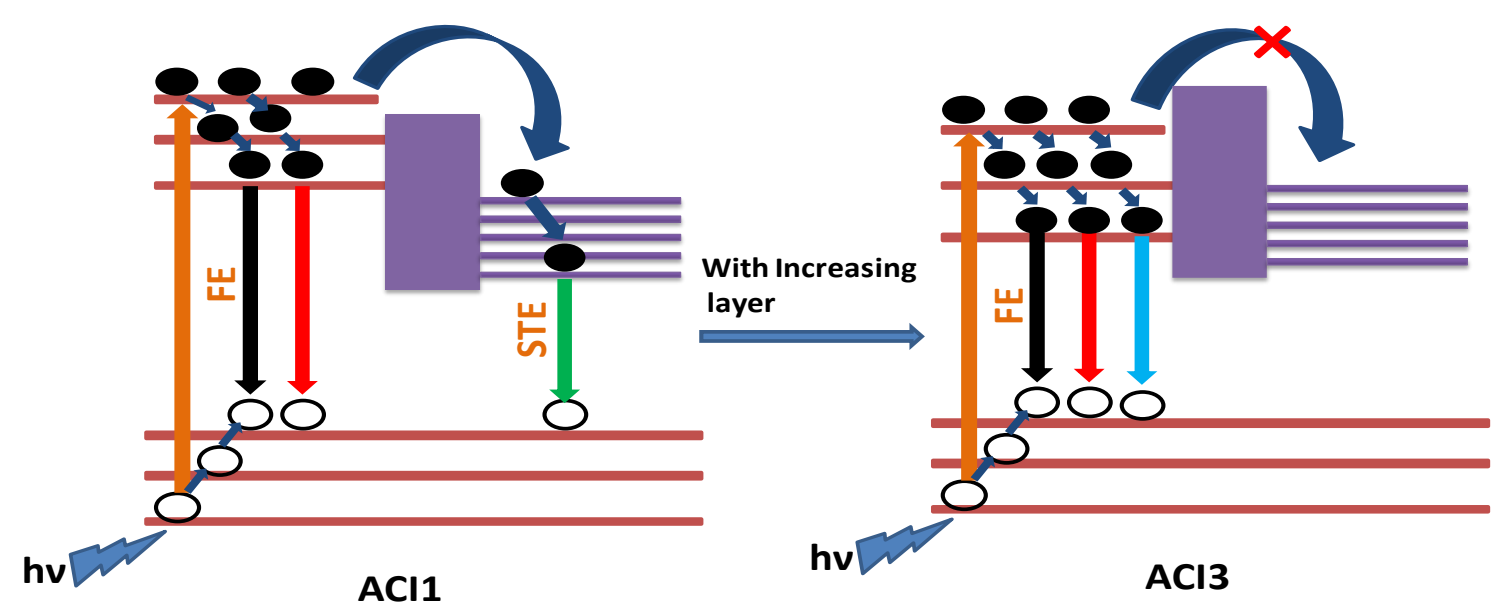

Scheme 2. Schematic illustration of the exciton dynamics in ACI perovskites following excitation with intensity $I 2$. The orange arrows indicate the pump-induced band-to-band transition, the dark blue arrows constitute the carrier relaxation to the band edge, the curved blue arrow designates the self-trapping of the exciton (STE), the black arrows stand for single exciton recombination and the red arrows for biexcitonic recombination, the light blue arrow indicates the higher-order Auger recombination, and the dark green arrow stands for the STE recombination. The energy barrier, which has to be overcome for the STE process is depicted as purple box, which schematically shows the decreased band gap and increased barrier height for ACI3.

After now having addressed the details of the exciton dynamics, we now discuss another aspect of the influence of the different 2D layers. The optical anisotropy plays an important role when it comes to the properties of a variety of optical components such as polarizers and 
waveplates. ${ }^{48-50} 2 \mathrm{D}$ layered structures in particular show inherently large optical anisotropies due to the high orientation of their crystal structures. ${ }^{43,48,51} 2 \mathrm{D}$ perovskites also exhibit a considerable exciton anisotropy between the crystallographic planes and cross planes, as these planes have different polarization orientations. ${ }^{43}$ The optical anisotropy can also be enhanced by artificially orientating the micro/nanostructures. ${ }^{52-54}$ These specially oriented structures can lead to unnecessary optical losses and at the same time to a more difficult fabrication entailing higher costs. ${ }^{48,} 50$ In our case, samples have been prepared without specially designing an anisotropic morphology. We have investigated the anisotropy properties of the different 2D ACI perovskites with varying layers described above.

For this, we have used polarized pump-probe spectroscopy. In these experiments, we excited the samples with linearly polarized pump pulses above the optical bandgap and probed the dynamical evolution of anisotropy with linearly polarized probe pulses as a function of delay time. We selectively recorded the dynamics probed with laser pulses having polarizations aligned parallel or perpendicular to the linear polarization of the pump pulses directly after the samples. The probe transmission through the sample with $\left(T_{\text {pump-on }}\right)$ and without $\left(T_{\text {pump-off }}\right)$ the pump pulses interacting with the samples is used to calculate the relative change in transmission following photoexcitation $\Delta T / T=\left(T_{\text {pump-on }}-T_{\text {pumpoff }}\right) / T_{\text {pump-off. }}$ This method is sensitive to the coupling between the optical polarization vector of the absorbed light and the transition dipole matrix element of the electronic states, which allows us to probe optical anisotropies in the excited-state population. In general, the transient absorption anisotropy provides valuable insights into the polarization memory loss of the electronic states, which is due to the reorientation of the excited state species or their diffusion to a comparatively lower energy site. The light-induced polarization and intrinsic dipole moment of the material results in an anisotropy, which can be calculated using the following equation: ${ }^{36,55}$

$$
r(t)=\frac{(\Delta T / T) \|-(\Delta T / T) \perp}{(\Delta T / T) \|+2 \times(\Delta T / T) \perp}
$$


Here, $(\Delta T / T) \|$ and $(\Delta T / T) \perp$ are the differential probe pulse transmissions for the probe pulse polarization parallel and perpendicular to that of the pump pulse, respectively. Figure 4 shows the anisotropy decay patterns for ACI1, ACI2, and ACI3. In the case of ACI3, the maximum anisotropy within the first picosecond is about $0.39 \pm 0.01$, which is equivalent to the previously reported dichroic ratio for $2 \mathrm{D}$ perovskites. ${ }^{51}$
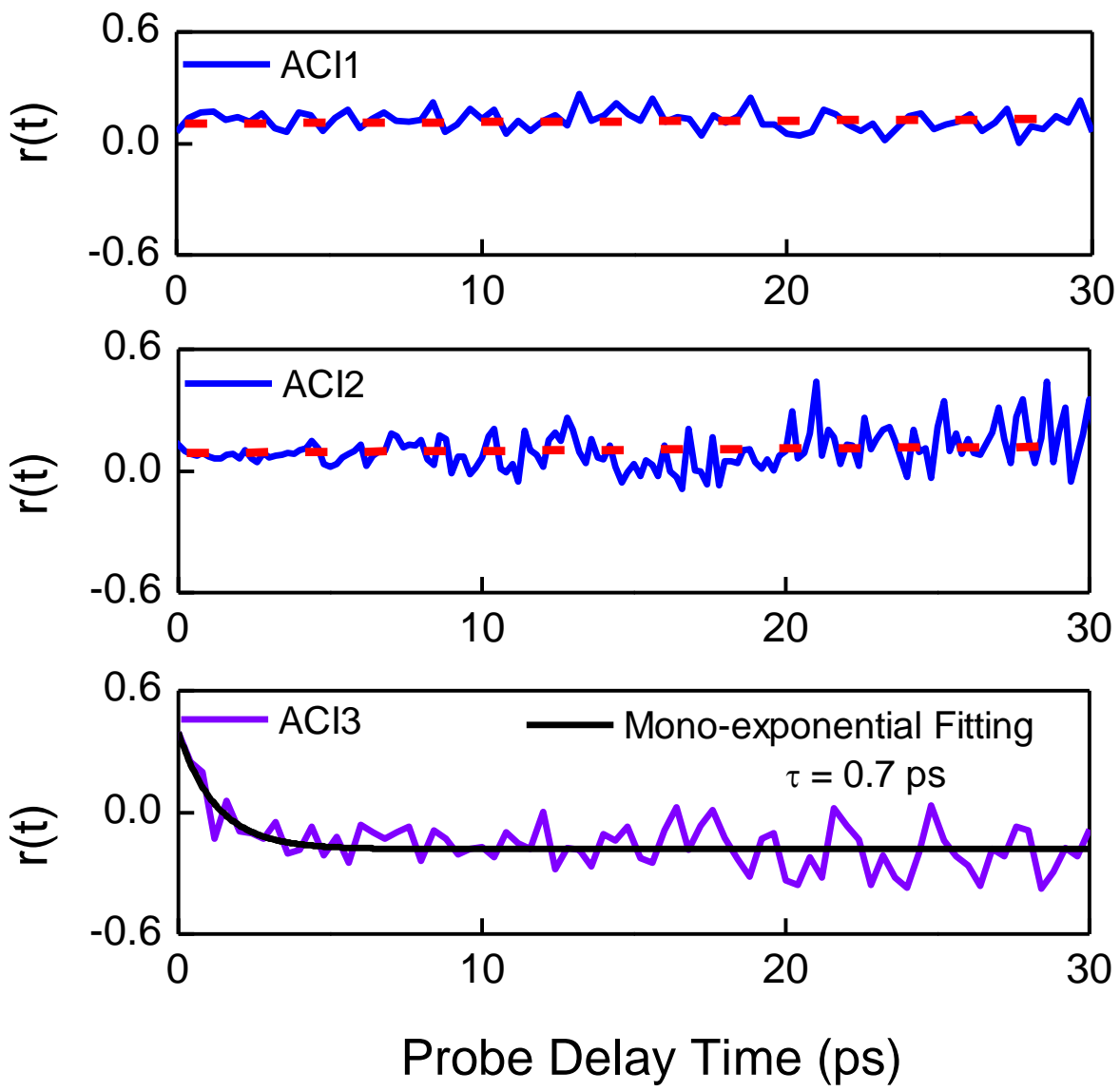

Figure 4. Polarization anisotropy decay dynamics of ACI1, ACI2, and ACI3. A constant photoinduced absorption background was offset before calculating the polarization anisotropy. Pump and probe wavelengths are comparable to those used for the TA dynamics measurements.

As a result, we find that the anisotropy decreases (at time zero) with decreasing layer (see Table 1). Fang et al. ${ }^{51}$ reported that the polarization orientation in $2 \mathrm{D}$ perovskite is parallel to the corrugated inorganic layers. Furthermore, from GIWAXS measurements we observe that the 
crystal structure of ACI3 is more ordered than for ACI2 and ACI1. This indicates that ACI1 contains differently oriented crystal plane whereas with increasing $n$, the orientation of the crystal plane becomes more homogeneous. Recently, Li and co-workers reported that FE and STE show a completely opposite polarization-dependent behavior; the nature of the excitons depends on the orientation of the crystal planes. ${ }^{43,51}$ As ACI3 contain more oriented crystal planes and high anisotropy, it can be concluded that ACI3 contains mainly one type of excitons (FE) whereas ACI1 contains different types (FE and STE).

Table 1. Anisotropy of different ACI perovskites

\begin{tabular}{|c|c|}
\hline Sample & Anisotropy, $r(t=0 \mathrm{ps})$ \\
\hline ACI1 & 0.1039 \\
\hline ACI2 & 0.1305 \\
\hline ACI3 & 0.3944 \\
\hline
\end{tabular}

\section{Conclusions}

In summary, we have investigated the exciton dynamics in a series of hybrid 2D perovskites with two different alternating cations in the interlayer space (ACI) with different numbers of layers ( $n=1,2$, and 3) using time-resolved photoluminescence, transient absorption, and transient anisotropy measurements. Our results demonstrate that the different order exciton recombination processes are affected by very fast self-trapping of excitons. The self-trapping of excitons increases with decreasing $n$, as the energy barrier between FE and STE states decreases. With increasing $n$, the energy barrier between the FE and STE states increases, and as a result, the higher-order recombination processes dominate over the self-trapping process. The time-resolved decay dynamics reveal that the average lifetime increases with decreasing layer thickness due to the increasing contribution of self-trapping of excitons. The study of the 
transient anisotropy shows that the crystal planes as well as the excitons become more oriented with increasing $n$. We believe that our study provides valuable information, which will be useful for the development of photovoltaic and polarization optical devices based on ACI perovskites.

\section{Supporting Information}

Absorption spectra of ACI1 and ACI2 perovskite, TA spectrum of ACI1 perovskite, fit of the PL spectrum of the ACI1 perovskite using a Voigt line profile, fits of the TA dynamics using two-body and three-body exciton recombination models, tabled parameters resulting from the GIWAXS analysis and fitting of the TA dynamics.

\section{Acknowledgements}

S.G is thankful to the Alexander von Humboldt Foundation, Germany for the award of a postdoctoral fellowship and financial support. J.H. acknowledges financial support from the Research Foundation-Flanders (FWO, Grant No. G983.19N, G0A5817N, and ZW15_09G0H6316N), the Flemish government through long-term structural funding Methusalem (CASAS2, Meth/15/04), the KU Leuven Research Fund (iBOF-21-085 PERSIST) and the MPI as MPI fellow. B.P acknowledges a postdoctoral fellowship from the Research FoundationFlanders (FWO Grant No. 1275521N). We also thankful for the funding received from the EUH2020 Research and Innovation Programme under grant agreement no. 654360 having benefitted from access to the Austrian SAXS beamline provided by TUG@Elettra in Trieste, Italy, within the framework of the NFFA-Europe Transnational Access Activity. 


\section{References}

1. Yuan, M.; Quan, L.N.; Comin, R.; Walters, G.; Sabatini, R.; Voznyy, O.; Hoogland, S.; Zhao, Y.; Beauregard, E.M.; Kanjanaboos, P.; et al., Perovskite Energy Funnels for Efficient Light-Emitting Diodes. Nat. Nanotechnol. 2016, 11, 872-877.

2. Manser, J. S.; Christians, J. A.; Kamat, P. V., Intriguing Optoelectronic Properties of Metal Halide Perovskites. Chem. Rev. 2016, 116, 12956-13008.

3. Stranks, S. D.; Snaith, H. J., Metal-Halide Perovskites for Photovoltaic and LightEmitting Devices. Nat. Nanotechnol. 2015, 10, 391.

4. Sum, T. C.; Mathews, N., Advancements in Perovskite Solar Cells: Photophysics Behind the Photovoltaics. Energy Environ. Sci. 2014, 7, 2518-2534.

5. Pradhan, B.; Kumar, G. S.; Sain, S.; Dalui, A.; Ghorai, U. K.; Pradhan, S. K.; Acharya, S., Size Tunable Cesium Antimony Chloride Perovskite Nanowires and Nanorods. Chem. Mater. 2018, 30, 2135-2142.

6. Tian, Y.; Peter, M.; Unger, E.; Abdellah, M.; Zheng, K.; Pullerits, T.; Yartsev, A.; Sundström, V.; Scheblykin, I. G., Mechanistic Insights into Perovskite Photoluminescence Enhancement: Light Curing with Oxygen Can Boost Yield Thousandfold. Phys. Chem. Chem. Phys. 2015, 17, 24978-24987.

7. Brenner, T. M.; Egger, D. A.; Kronik, L.; Hodes, G.; Cahen, D., Hybrid OrganicInorganic Perovskites: Low-Cost Semiconductors with Intriguing Charge-Transport Properties. Nat. Rev. Mater. 2016, 1, 15007.

8. Ghosh, S.; Shi, Q.; Pradhan, B.; Kumar, P.; Wang, Z.; Acharya, S.; Pal, S. K.; Pullerits, T.; Karki, K. J., Phonon Coupling with Excitons and Free Carriers in Formamidinium Lead Bromide Perovskite Nanocrystals. J. Phys. Chem. Lett. 2018, 9, 4245-4250. 
9. Protesescu, L.; Yakunin, S.; Bodnarchuk, M. I.; Bertolotti, F.; Masciocchi, N.; Guagliardi, A.; Kovalenko, M. V., Monodisperse Formamidinium Lead Bromide Nanocrystals with Bright and Stable Green Photoluminescence. J. Am. Chem. Soc. 2016, 138, 14202-14205. 10. Cai, B.; Li, X.; Gu, Y.; Harb, M.; Li, J.; Xie, M.; Cao, F.; Song, J.; Zhang, S.; Cavallo, L.; et al., Quantum Confinement Effect of Two-Dimensional All-Inorganic Halide Perovskites. Sci. China Mater. 2017, 60, 811-818.

11. Ghosh, S.; Pal, S. K.; Karki, K. J.; Pullerits, T., Ion Migration Heals Trapping Centers in $\mathrm{CH} 3 \mathrm{NH} 3 \mathrm{PbBr} 3$ Perovskite. ACS Energy Lett. 2017, 2, 2133-2139.

12. Trinh, M. T.; Wu, X.; Niesner, D.; Zhu, X.-Y., Many-Body Interactions in PhotoExcited Lead Iodide Perovskite. J. Mater. Chem. A 2015, 3, 9285-9290.

13. Ghosh, S.; Shi, Q.; Pradhan, B.; Mushtaq, A.; Acharya, S.; Karki, K. J.; Pullerits, T.; Pal, S. K., Light Induced Defect Healing and Strong Many-Body Interactions in Formamidinium Lead Bromide Perovskite Nanocrystals. J. Phys. Chem. Lett. 2020, 11, 12391246.

14. Wu, X.; Trinh, M. T.; Niesner, D.; Zhu, H.; Norman, Z.; Owen, J. S.; Yaffe, O.; Kudisch, B. J.; Zhu, X.-Y., Trap States in Lead Iodide Perovskites. J. Am. Chem. Soc. 2015, 137, 20892096.

15. deQuilettes, D. W.; Zhang, W.; Burlakov, V. M.; Graham, D. J.; Leijtens, T.; Osherov, A.; Bulović, V.; Snaith, H. J.; Ginger, D. S.; Stranks, S. D., Photo-Induced Halide Redistribution in Organic-Inorganic Perovskite Films. Nat. Commun. 2016, 7, 11683.

16. Becker, M. A.; Vaxenburg, R.; Nedelcu, G.; Sercel, P. C.; Shabaev, A.; Mehl, M. J.; Michopoulos, J. G.; Lambrakos, S. G.; Bernstein, N.; Lyons, J. L., Bright Triplet Excitons in Caesium Lead Halide Perovskites. Nature 2018, 553, 189.

17. Zuo, L.; Guo, H.; deQuilettes, D. W.; Jariwala, S.; De Marco, N.; Dong, S.; DeBlock, R.; Ginger, D. S.; Dunn, B.; Wang, M., Polymer-Modified Halide Perovskite Films for Efficient and Stable Planar Heterojunction Solar Cells. Sci. Adv. 2017, 3, e1700106. 
18. Meng, L.; You, J.; Yang, Y., Addressing the Stability Issue of Perovskite Solar Cells for Commercial Applications. Nature Commun. 2018, 9, 1-4.

19. Soe, C. M. M.; Stoumpos, C. C.; Kepenekian, M.; Traoré, B.; Tsai, H.; Nie, W.; Wang, B.; Katan, C.; Seshadri, R.; Mohite, A. D., New Type of 2d Perovskites with Alternating Cations in the Interlayer Space, $(\mathrm{C}(\mathrm{NH} 2) 3)(\mathrm{CH} 3 \mathrm{NH} 3) n \mathrm{PbnI} 3 \mathrm{n}+1$ : Structure, Properties, and Photovoltaic Performance. J. Am. Chem. Soc. 2017, 139, 16297-16309.

20. Kim, H.; Huynh, K. A.; Kim, S. Y.; Le, Q. V.; Jang, H. W., 2D and Quasi-2D Halide Perovskites: Applications and Progress. Phys. Status Solidi RRL 2019, 1900435.

21. Zheng, K.; Pullerits, T., Two Dimensions Are Better for Perovskites. J. Phys. Chem. Lett. 2019, 10, 5881.

22. Chen, Y.; Sun, Y.; Peng, J.; Zhang, W.; Su, X.; Zheng, K.; Pullerits, T.; Liang, Z., Tailoring Organic Cation of 2D Air-Stable Organometal Halide Perovskites for Highly Efficient Planar Solar Cells. Adv. Energy Mater. 2017, 7, 1700162.

23. Yuan, H.; Debroye, E.; Janssen, K.; Naiki, H.; Steuwe, C.; Lu, G.; Moris, M., L.; Orgiu, E.; Uji-I, H.; De Schryver, F. J. Phys. Chem. Lett. 2016, 7, 561.

24. Huo, C.; Cai, B.; Yuan, Z.; Ma, B.; Zeng, H., Two-Dimensional Metal Halide Perovskites: Theory, Synthesis, and Optoelectronics. Small Methods 2017, 1, 1600018.

25. Ruddlesden, S.; Popper, P., The Compound Sr3Ti2O7 and Its Structure. Acta Crystallogr. 1958, 11, 54-55.

26. Tsai, H.; Nie, W.; Blancon, J.-C.; Stoumpos, C. C.; Asadpour, R.; Harutyunyan, B.; Neukirch, A. J.; Verduzco, R.; Crochet, J. J.; Tretiak, S., High-Efficiency Two-Dimensional Ruddlesden-Popper Perovskite Solar Cells. Nature 2016, 536, 312-316.

27. Cheng, P.; Xu, Z.; Li, J.; Liu, Y.; Fan, Y.; Yu, L.; Smilgies, D.-M.; Müller, C.; Zhao, K.; Liu, S. F., Highly Efficient Ruddlesden-Popper Halide Perovskite PA2MA4Pb5I16 Solar Cells. ACS Energy Lett. 2018, 3, 1975-1982. 
28. Dion, M.; Ganne, M.; Tournoux, M., Nouvelles Familles De Phases $\mathrm{M}^{\mathrm{I}} \mathrm{M}^{\mathrm{II}}{ }_{2} \mathrm{Nb}_{3} \mathrm{O}_{10}$ a Feuillets "Perovskites". Mater. Res. Bull. 1981, 16, 1429-1435.

29. Jacobson, A.; Johnson, J. W.; Lewandowski, J., Interlayer Chemistry between Thick Transition-Metal Oxide Layers: Synthesis and Intercalation Reactions of K[Ca2Nan3NbnO3n+ 1](3.Itoreq.n.Itoreq.7). Inorg. Chem. 1985, 24, 3727-3729.

30. Mao, L.; Ke, W.; Pedesseau, L.; Wu, Y.; Katan, C.; Even, J.; Wasielewski, M. R.; Stoumpos, C. C.; Kanatzidis, M. G., Hybrid Dion-Jacobson 2D Lead Iodide Perovskites. J. Am. Chem. Soc. 2018, 140, 3775-3783.

31. Zhang, Y.; Wang, P.; Tang, M.-C.; Barrit, D.; Ke, W.; Liu, J.; Luo, T.; Liu, Y.; Niu, T.; Smilgies, D.-M., Dynamical Transformation of Two-Dimensional Perovskites with Alternating Cations in the Interlayer Space for High-Performance Photovoltaics. J. Am. Chem. Soc. 2019, $141,2684-2694$.

32. Luo, T.; Zhang, Y.; Xu, Z.; Niu, T.; Wen, J.; Lu, J.; Jin, S.; Liu, S.; Zhao, K., Compositional Control in 2d Perovskites with Alternating Cations in the Interlayer Space for Photovoltaics with Efficiency over 18\%. Adv. Mater. 2019, 31, 1903848.

33. Ni, L.; Huynh, U.; Cheminal, A.; Thomas, T. H.; Shivanna, R.; Hinrichsen, T. F.; Ahmad, S.; Sadhanala, A.; Rao, A., Real-Time Observation of Exciton-Phonon Coupling Dynamics in Self-Assembled Hybrid Perovskite Quantum Wells. ACS Nano 2017, 11, 1083410843.

34. Zheng, K.; Chen, Y.; Sun, Y.; Chen, J.; Chábera, P.; Schaller, R.; Al-Marri, M. J.; Canton, S. E.; Liang, Z.; Pullerits, T., Inter-Phase Charge and Energy Transfer in RuddlesdenPopper 2D Perovskites: Critical Role of the Spacing Cations. J. Mater. Chem. A 2018, 6, 62446250.

35. Khan, T. Z.; Donfack, P.; Namboodiri, M.; Kazemi, M. M.; Bom, S.; Wagner, V.; Materny, A., Femtosecond Time-Resolved Transient Absorption Spectroscopy with Sub- 
Diffraction-Limited Spatial Resolution Reveals Accelerated Exciton Loss at Gold-Poly (3Hexylthiophene) Interface. J. Phys. Chem. C 2018, 122, 3454-3462.

36. Rana, D.; Donfack, P.; Jovanov, V.; Wagner, V.; Materny, A., Ultrafast Polaron-Pair Dynamics in a Poly (3-Hexylthiophene-2, 5-Diyl) Device Influenced by a Static Electric Field: Insights into Electric-Field-Related Charge Loss. Phys. Chem. Chem. Phys. 2019, 21, 2123621248.

37. Tokunaga, A.; Uriarte, L. M.; Mutoh, K.; Fron, E.; Hofkens, J.; Sliwa, M.; Abe, J., Photochromic Reaction by Red Light Via Triplet Fusion Upconversion. J. Am. Chem. Soc. 2019, 141, 17744-17753.

38. Amenitsch, H.; Rappolt, M.; Kriechbaum, M.; Mio, H.; Laggner, P.; Bernstorff, S., First Performance Assessment of the Small-Angle X-Ray Scattering Beamline at Elettra. J. Synchrotron Radiat. 1998, 5, 506-508.

39. Burian, M.; Meisenbichler, C.; Naumenko, D.; Amenitsch, H., Saxsdog: Open Software for Real-Time Azimuthal Integration of 2D Scattering Images. arXiv preprint arXiv:2007.02022 2020.

40. Jiang, Z., Gixsgui: A Matlab Toolbox for Grazing-Incidence X-Ray Scattering Data Visualization and Reduction, and Indexing of Buried Three-Dimensional Periodic Nanostructured Films. J. Appl. Crystallogr. 2015, 48, 917-926.

41. Wright, A. D.; Verdi, C.; Milot, R. L.; Eperon, G. E.; Pérez-Osorio, M. A.; Snaith, H. J.; Giustino, F.; Johnston, M. B.; Herz, L. M., Electron-Phonon Coupling in Hybrid Lead Halide Perovskites. Nat. Commun. 2016, 7, 11755.

42. Smith, M. D.; Jaffe, A.; Dohner, E. R.; Lindenberg, A. M.; Karunadasa, H. I., Structural Origins of Broadband Emission from Layered Pb-Br Hybrid Perovskites. Chem. Sci. 2017, 8, 4497-4504.

43. Li, J.; Ma, J.; Cheng, X.; Liu, Z.; Chen, Y.; Li, D., Anisotropy of Excitons in TwoDimensional Perovskite Crystals. ACS Nano 2020, 14, 2156-2161. 
44. Song, K.; Williams, R., Self-Trapped Excitons, Springer Series in Solid State Sciences, Vol. 105, Springer-Verlag, Berlin 1993.

45. Li, J.; Wang, H.; Li, D., Self-Trapped Excitons in Two-Dimensional Perovskites. Front. Optoelectron. 2020, 13, 225-234.

46. Jin, H.; Debroye, E.; Keshavarz, M.; Scheblykin, I. G.; Roeffaers, M. B.; Hofkens, J.; Steele, J. A., It's a Trap! On the Nature of Localised States and Charge Trapping in Lead Halide Perovskites. Mater. Horiz. 2020, 7, 397-410.

47. Deng, X.; Wen, X.; Huang, S.; Sheng, R.; Harada, T.; Kee, T. W.; Green, M.; HoBaillie, A., Ultrafast Carrier Dynamics in Methylammonium Lead Bromide Perovskite. J. Phys. Chem. C 2016, 120, 2542-2547.

48. Liu, F.; Zheng, S.; He, X.; Chaturvedi, A.; He, J.; Chow, W. L.; Mion, T. R.; Wang, X.; Zhou, J.; Fu, Q.; et al., Highly Sensitive Detection of Polarized Light Using Anisotropic 2D ReS2. Adv. Funct. Mater. 2016, 26, 1169-1177.

49. Lu, W. G.; Wu, X. G.; Huang, S.; Wang, L.; Zhou, Q.; Zou, B.; Zhong, H.; Wang, Y., Strong Polarized Photoluminescence from Stretched Perovskite-Nanocrystal-Embedded Polymer Composite Films. Adv. Opt. Mater. 2017, 5, 1700594.

50. Niu, S.; Joe, G.; Zhao, H.; Zhou, Y.; Orvis, T.; Huyan, H.; Salman, J.; Mahalingam, K.; Urwin, B.; Wu, J.; et al., Giant Optical Anisotropy in a Quasi-One-Dimensional Crystal. Nat. Photonics 2018, 12, 392-396.

51. Fang, C.; Xu, M.; Ma, J.; Wang, J.; Jin, L.; Xu, M.; Li, D., Large Optical Anisotropy in Two-Dimensional Perovskite $[\mathrm{CH}(\mathrm{NH} 2) 2][\mathrm{C}(\mathrm{NH} 2) 3] \mathrm{PbI} 4$ with Corrugated Inorganic Layers. Nano Lett. 2020, 20, 2339-2347.

52. Kats, M. A.; Genevet, P.; Aoust, G.; Yu, N.; Blanchard, R.; Aieta, F.; Gaburro, Z.; Capasso, F., Giant Birefringence in Optical Antenna Arrays with Widely Tailorable Optical Anisotropy. Proc. Natl. Acad. Sci. U.S.A 2012, 109, 12364-12368. 
53. Yu, N.; Capasso, F., Flat Optics with Designer Metasurfaces. Nature Mater. 2014, 13, 139-150.

54. Yang, S.-H.; Cooper, M. L.; Bandaru, P. R.; Mookherjea, S., Giant Birefringence in Multi-Slotted Silicon Nanophotonic Waveguides. Opt. Express 2008, 16, 8306-8316.

55. Rivett, J. P.; Tan, L. Z.; Price, M. B.; Bourelle, S. A.; Davis, N. J.; Xiao, J.; Zou, Y.; Middleton, R.; Sun, B.; Rappe, A. M.; et al., Long-Lived Polarization Memory in the Electronic States of Lead-Halide Perovskites from Local Structural Dynamics. Nat. Commun. 2018, 9, 3531. 